\title{
"Sick" MRI syndrome
}

\section{J Kushner}

\section{At this time, MRI is a better indicator of tissue outcome rather than clinical destiny}

pli he report by Marx et al (this issue, pp572-575 $)^{1}$ joins the ever growing literature dealing with multiparametric MRI studies of tissue characteristics in stroke. Markers of ischaemic perturbations of cell homoeostasis and perfusion, including changes in diffusion and perfusion weighted imaging (DWI and PWI), seem now to allow human stroke studies more analogous to animal models. Although debate rages, current optimism maintains that MRI delineates the three main tissue compartments in ischaemia. Conceptually these compartments are (a) irreversibly damaged tissue, (b) minimally disrupted tissue destined for eventual recovery, and (c) the "penumbra" or "borderzone" or "at-risk" region where outcome hangs in the balance between irrevocable damage and some degree of recovery, and which may be amenable to treatment.

Recent human data suggest that DWI and DWI-PWI mismatches strongly predict tissue outcome after ischaemia. Despite this progress in neuroimaging, clinical and clinicopathological MRI correlation has lagged behind. Reconciling recent MRI studies of stroke with each other is not straightforward, and it is also daunting to relate them to previous investigations of stroke using other reliable structural and haemometabolic techniques. ${ }^{45}$ To date, human MRI studies have used many different paradigms, and this situation stands in contrast with standardised models found in the animal stroke literature. Variations in clinical criteria, imaging protocols, and myriad other factors introduce obvious confounding influences in these studies, and it should be assumed that lack of standardised approaches can only increase exposure to not so obvious bias.

Marx et al report a relatively high incidence of DWI changes following posterior circulation ischaemia where the clinical syndrome actually was transient or reversible. DWI alterations tended to be more prominent with longer lasting ischaemic symptoms yet were detected despite ultimate clinical recovery. This bears mention, indeed, for one would expect the observed DWI changes to bode ill when in actuality full recovery was the case. This represents a potential quandary: how should one reconcile a "sick" MRI with a healthy patient? A simple explanation may be that, at this time, MRI is a better indicator of tissue outcome rather than clinical destiny. If future reports invoke MRI as an indicator of clinical outcome, or as a marker of the impact of therapeutic intervention, then data will be needed across the full spectrum of stroke severity from fixed deficits to transient syndromes. Standardised study designs are needed so that the true utility of functional MRI is established in both natural history and interventional studies of stroke. Consensus on this score would be useful now, for the widespread availability of MRI, in contrast with other techniques such as PET or SPECT, suggests that this literature will continue to proliferate.

While this field remains in flux, clinical neurology should take note. At a minimum, these MRI markers of ischaemia must be regarded as new harbingers in the menagerie threatening haemometabolic risk, and attention must be paid. Their appearance should spur the clinician to redouble efforts to apply available medical and surgical treatments, whether they be acute, or preemptive and prophylactic, to address treatable haemorheological and haemodynamic abnormalities.

J Neurol Neurosurg Psychiatry 2002;72:564

\section{Author's affiliations}

M J Kushner, Eastern Carolina University School of Medicine and the Wilson Neurology Center, Wilson, North Carolina, USA

\section{REFERENCES}

1 Marx JJ, Mike-Gruettner A, Thoemke F, Fitzek S, Fitzek C, Vucurevic G Urban PP, Stoeter P, Hopf HC. Diffusion weighted magnetic resonance imaging in the diagnosis of reversible ischaemic deficits of the brainstem. J Neurol Neurosurg Psychiatry 2002:72.572-575.

2 Astrup J, Siesjo BK, Symon L. Threshold in cerebral ischemia: the ischemic penumbra. Stroke 1981;12:723-5.

3 Baird AE, Benfield A, Schlaug G, et al. Enlargement of human cerebral ischemic lesion volumes measured by diffusion-weight magnetic imaging. Ann Neurol 1997;41:581-9.

4 Baron JC, Frackowiak R, Herholz K, et al. Use of PET methods for measurement of cerebral energy metabolism and hemodynamics in cerebrovascular disease. $J$ Cereb Blood Flow Metab 1989:9:723-42.

5 Fieschi C, Argentino C, Lenzi GL, et al. Clinical and experimental evaluation of patients with ischemic stroke within the first six hours. J Neurol Sci 1989;91:311-22.

\section{White matter change and stroke risk}

\section{P M Matthews}

in rge cohort studies have defined an increasing range of therapeutic interventions that can be applied to reduce stroke risk. However, application of these in a specialist practice is complicated by the problem of interindividual variation.
A major practical issue is to differentiate those patients who will do well on standard treatment from those for whom a specifically targeted, more aggressive approach is needed. Ideally, it would be possible to profile patients at presentation to identify those at highest risk. If such a strategy could be made reliable, it would help simultaneously to balance a commitment to provide the best care available for each patient and to reassure government and other funders that potentially expensive therapeutic interventions are being employed in the most cost-effective fashion.

MRI provides an attractive tool for stratifying risk in a stroke population. It offers a good definition of brain injury and promises to deliver increasingly quantitative markers of specific pathological changes. ${ }^{1}$ With growing access to MRI scanners and routine imaging of stroke cases, even this relatively expensive "screening test" would be of potential 
value as a marker of risk if it can be shown to be both sensitive and prognostically useful. A fundamental problem that has thus far limited enthusiasm for the use of MRI in establishing prognosis (as opposed to establishing clinical-pathological correlations) has been the non-specificity of T2 hyperintensity-the most sensitive marker-on conventional MRI. T2 hyperintensity in white matter, for example, can arise from enlarged perivascular spaces, increased tissue fluid, inflammation, or gliosis.

In the paper by Yamauchi et al ${ }^{2}$ (this issue pp576-82) there are important new observations suggesting that conventional T2 weighted MRI may provide clinically useful information for risk stratification in an older stroke population. This paper follows a series of communications from several groups showing correlations between recognised risk factors such as hypertension ${ }^{3}$ and clinical syndromes such as cognitive impairment. ${ }^{4}$

Yamauchi et al ${ }^{2}$ followed prospectively 89 patients who either had symptomatic lacunar infarcts or who were neurologically normal and presented for other reasons. Subjects with large vessel disease were specifically excluded. White matter lesions were graded using a simple scoring system based on the extent and distribution of changes. Clinical monitoring and serial MRI studies were performed over a period of around four years to test the predictive value of white matter lesion changes at baseline and over time.

Seven patients had ischaemic or haemorrhagic strokes. These occurred only in patients with white matter lesions at baseline, and the proportion of patients who had stroke was greater in those with more severe white matter lesion changes than in those with milder white matter lesions. A Cox proportional hazards model identified both the expected risk factors for stroke (hypertension, uncontrolled diabetes mellitus, and smoking) and also the extent of white matter lesion changes. However, in a multiparametric model, the only independent predictors of stroke were the extent of the white matter lesions and uncontrolled diabetes mellitus. When patients with lacunar infarcts were considered separately, only the extent of white matter lesion changes was an independent predictor of later stroke.

Over the follow up period none of the patients showed regression of white matter lesions, suggesting irreversible pathology. Extending the association of white matter lesion extent with stroke risk found in the cross sectional studies, patients showing increases in white matter lesion load during the period of observation had a greater risk of later stroke.

The risk of stroke increases substantially after an initial cerebrovascular event. However, it has been less clear whether white matter changes can be interpreted as "silent strokes" that similarly contribute to risk. This study persuasively supports the notion that the extent of white matter lesion changes is an independent predictor and may provide prognostic information. Further work is needed to confirm these findings. Potential areas for such further work include the application of objective criteria for evaluation, the use of whole brain imaging, and thinner slices to improve the definition of changes. Nonetheless, Yamauchi et al offer the gratifying prospect of better targeting of aggressive management, although clearly a substantial therapeutic challenge remains-a disappointing observation in their study was that the severe white matter lesion group continued to have a higher risk of stroke despite apparently appropriate conventional management of vascular factors during the follow up period. Greater future effort might be directed towards developing therapeutic strategies directed at high risk populations, defined jointly by clinical and imaging measures.

J Neurol Neurosurg Psychiatry 2002;72:564-565

\section{Author's affiliations}

P M Matthews, Centre for Functional MRI of the Brain, John Radcliffe Hospital, Headley Way, Headington, Oxford OX3 9DU, UK; paul@fmrib.ox.ac.uk

\section{REFERENCES}

1 Matthews PM, De Stefano N, Fazekas F, et al. The future of magnetic resonance-based techniques in neurology. Eur J Neurol 2001;8:17-25.

2 Yamaychi H, Fukuda H, Oyanaki C. Significance of white matter high intensity lesions as a predictor of stroke from arteriosclerosis. J Neurol Neurosurg Psychiatry 2002;72:576-82.

3 de Leeuw LE, de Groot JC, Oudkerk M, et al. A follow up study of blood pressure and cerebral white matter lesions. Ann Neurol 1999;46:827-33.

4 de Groot JC, de Leeuw FE, Oudkerk M, et al. Cerebral white matter lesions and cognitive function: the Rotterdam Scan Study. Ann Neurol 2000;47: 145-51

\section{Qualitative performance characteristics differentiate dementia with Lewy bodies and Alzheimer's disease}

\section{Ballard, J O'Brien, M Tovee}

New approach to assessment of patients with dementia with Lewy bodies is clinically useful in differentiating them from patients with Alzheimer's disease

A ccurate and early clinical diagnosis of dementia with Lewy bodies (DLB) is essential for optimal management so that pitfalls such as severe neuroleptic sensitivity ${ }^{1}$ can be avoided, a trial of cholinesterase treatment can be instigated, ${ }^{2}$ and parkinsonian symptoms ${ }^{3}$ can be appropriately treated, weighing up the balance of psychiatric and motor symptoms and fall risk. In practice it has often been difficult to differentiate DLB from other forms of dementia, particularly Alzheimer's disease. Several previous studies have suggested that DLB and Alzheimer's disease can be differentiated on the basis of visual and visuospatial tasks. ${ }^{45}$ The tasks most easily applicable to initial diagnosis are drawing tests (such as the clock drawing). ${ }^{6}$ However, this approach ignores what is one of the more striking features of DLB: fluctuating cognition. Operationalised criteria for the clinical diagnosis of DLB (which do consider a broad range of symptoms) have achieved high levels of specificity $(>90 \%)^{7}$; in many studies sensitivity has been as low as $50 \%{ }^{8}$ Probably the major obstacle to accurate diagnosis has been the difficulty of identifying fluctuating cognition; for example, two inter-rater reliability studies have indicated that agreement 
between expert clinicians is barely better than chance. ${ }^{910}$

A body of work focusing on these issues has validated two clinical rating scales ${ }^{11}$ and indicated that fluctuation of reaction time on attentional tasks ${ }^{12}$ and variability in spectral frequency assessed by electroencephalography ${ }^{12}$ can also be useful methods for identifying and quantifying fluctuating cognition, which seems to be closely linked to impairments of consciousness.

In the paper by Doubleday et $a l^{13}$ (this issue, pp602-607) tackles these issues in an innovative way. The authors developed a standardised qualitative instrument based on observations of the patient during the course of the testing session. Information is obtained regarding a number of helpful domains including general inattention, distraction, and intrusions. Of particular interest, general inattention, distractibility, and environmentally cued intrusions were all significantly more common in DLB than in Alzheimer's disease, the latter occurring only in patients with DLB. In addition to the potential diagnostic value, this may be helpful in facilitating an understanding of the types of processing errors that are prominent in these patients. Perseveration and mental set shifting were also significantly more impaired in patients with DLB and they were more likely to confabulate.

This assessment method has been used as part of the standard diagnostic protocol that has achieved a high level of accuracy in 200 people with dementia at postmortem examination, although it is unclear how many of these people had neuropathologically confirmed DLB. Further work is needed to examine the inter-rater reliability and concurrent validity of the instrument, and comparisons between DLB and other dementias would also be helpful. In particular, patients with vascular dementia often experience some degree of fluctuating cognition and may be more prone to difficulties with set shifting and perseveration. Diagnostic discrimination between DLB and vascular dementia can be difficult and it would be useful to know whether the proposed assessment method can also contribute to this diagnostic issue. Therefore, although the method needs to be developed a little more, the work presents a new and useful approach that is very clinically applicable and that may be of considerable value in the assessment of patients with DLB.

\section{J Neurol Neurosurg Psychiatry}

\section{2;72:565-566}

\section{Authors' affiliations}

C Ballard, J O'Brien, M Tovee, Institute for Ageing and Health, Wolfson Research Centre, Newcastle upon Tyne, UK

Correspondence to: Professor C Ballard, Wolfson Research Centre, Institute for Ageing and Health, Newcastle General Hospital, Westgate Road, Newcastle upon Tyne NE4 6BE, UK; c.g.ballard@newcastle.ac.uk

\section{REFERENCES}

1 Ballard C, Grace J, McKeith I, et al. Neuroleptic sensitivity in dementia with Lewy bodies and Alzheimer's disease. Lancet 1998;351:1032-3.
2 McKeith I, Del Ser T, Spano P, et al. Efficacy of rivastigmine in dementia with Lewy bodies: a randomised, double blind, placebo controlled international study. Lancet 2000;356:2031-6.

3 Ganalingham KK, Byrne EJ, Thornton A, et al. Motor and cognitive function in Lewy body dementia: comparison with Alzheimer's and dementia: comparison with Alzheimer's and Psychiatry 1997;62:243-52.

4 Mori E, Shimomura T, Fujimori $M$, et al. Visuoperceptual impairment in dementia with Lewy bodies. Arch Neurol 2000:57:489-93.

5 Calderon J, Perry RJ, Erzinclioglu SW, et al. Perception, attention and working memory are disproportionately impaired in dementia with Lewy bodies compared with Alzheimer's disease. J Neurol Neurosurg Psychiatry 2001;70:157-64.

6 Ganalingham KK, Byrne EJ, Thornton A, et al. Clock drawing to differentiate Lewy body and Alzheimer type dementia syndrome. Lancet 1996;347:696-7.

7 McKeith IG, Ballard CG, Perry RH, et al. Prospective validation of the consensus criteria for the clinical diagnosis of dementia with Lewy bodies. Neurology 2000;54:1050-8.

8 McKeith IG, O'Brien J, Ballard C. Diagnosing dementia with Lewy bodies. Lancet 1999;354:1227-8.

9 Mega MS, Masterman DL, Benson DF, et al. Dementia with Lewy bodies: reliability and validity of clinical and pathologic criteria. Neurology 1996;47: 1403-9.

10 Litvan I, Maclntyre A, Goetz CG, et al. Accuracy of the clinical diagnoses of Lewy body disease, Parkinson disease, and dementia with Lewy bodies: a clinicopathologic study. Arch Neurol 1998;55:969-78.

11 Walker MP, Ayre GA, Cummings JL, et al. The clinician assessment of fluctuation and the one day fluctuation assessment scale: two methods to assess fluctuating confusion in methods to assess fluctuating confusion in
dementia. Br J Psychiatry 2000;177:252-6.

12 Walker MP, Ayre GA, Cummings JL, et al. Quantifying fluctuation in dementia with Lewy bodies, Alzheimer's disease and vascular dementia. Neurology 2000;54:1616-25.

13 Doubleday EK, Snowden JS, Varma AR, et al. Qualitative performance characteristics differentiate dementia with Lewy bodies and Alzheimer's disease. J Neurol Neurosurg Psychiatry 2002;72:602-7.

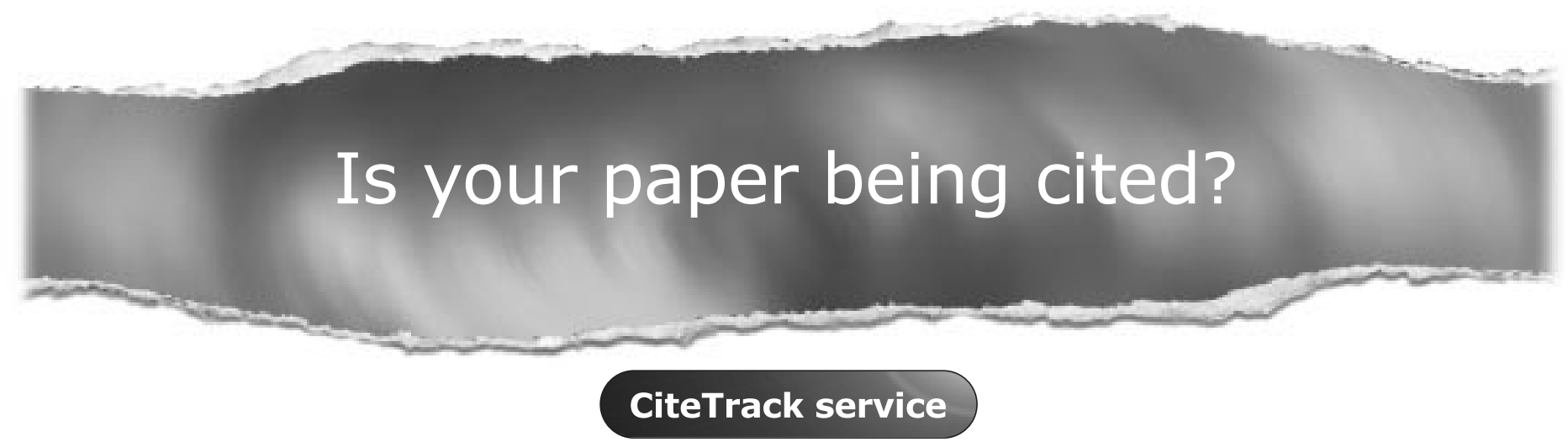

CiteTrack will alert you by email whenever new content in the Journal of Neurology, Neurosurgery, and Psychiatry or a participating journal is published that matches criteria you want to track Topics: Tell CiteTrack which words or subjects to watch for in new content Authors: Be alerted whenever key authors you are following publish a new paper Articles: Know whenever a paper of interest to you is referenced by another paper 